\title{
Topological Conditions for Wireless Control Networks
}

\author{
Miroslav Pajic
}

\author{
Shreyas Sundaram
}

\author{
George J. Pappas
}

\author{
Rahul Mangharam
}

\begin{abstract}
We study the problem of stabilizing a linear system over a wireless control network. We propose a scheme where each wireless node maintains a scalar state, and periodically updates it as a linear combination of neighboring plant outputs and node states. We make connections to decentralized fixed modes and structured system theory to provide conditions on the network topology that allow the system to be stabilized. Our analysis provides the minimal number of feedback edges that have to be introduced to stabilize the system over a network, and shows that as long as the network connectivity is larger than the geometric multiplicity of any unstable eigenvalue, stabilizing controllers can be constructed at each actuator. A byproduct of our analysis is that by co-designing the network dynamics with the controllers, delays in the network are not a factor in stabilizing the system.
\end{abstract}

\section{INTRODUCTION}

The widespread availability of low-cost wireless networking technology promises to bring about a shift in the architecture of industrial control systems. However, the introduction of wireless communications into the feedback loop also presents several challenges for real-time feedback control. For instance, delays due to multi-hop routing or packet dropouts due to transmission collisions can be detrimental to the goal of maintaining stability of the system. Substantial research has been devoted to understanding the limitations of performance in such settings (e.g., [1], [2], [3], [4]).

In a recent paper [5], we asked the following question: is it possible to formulate a distributed algorithm for the resource-constrained wireless nodes to follow so that the computation of the control law is done collectively by the network (rather than by a specially designated controller)? To answer this question, we considered a setup where each node maintains a limited internal state, and periodically updates it as a linear combination of the node states and plant outputs in its immediate neighborhood. We referred to this paradigm as a Wireless Control Network (WCN). The proposed scheme has several benefits, including easy scheduling of wireless transmissions, allowing compositional design, and its ability to handle geographically separated sensors and actuators.

In this paper, we continue our investigation of how the dynamics and topology of the wireless network can be leveraged to stabilize large scale plants. To do this, we make connections with the idea of decentralized fixed modes [6], [7], [8], [9], and with structured system theory, which allows us to use graph-theoretic tools to analyze linear

This research has been partially supported by the NSF-CNS 0931239 , NSF-MRI Grant 0923518 and by a grant from NSERC.

M. Pajic, G. J. Pappas and R. Mangharam are with the Department of Electrical and Systems Engineering, University of Pennsylvania, Philadelphia, PA, USA 19014. Email: \{pajic, pappasg, rahulm\} @seas.upenn.edu. S. Sundaram is with the Department of Electrical and Computer Engineering, University of Waterloo, Waterloo, ON, Canada, N2L 3G1. Email: ssundara@uwaterloo.ca. systems [10], [11], [12]. Specifically, we develop a method to incorporate structural analysis (which abstracts away all numerical system parameters) into the design of a control network for a numerically specified plant (potentially with eigenvalues of multiplicity larger than one); to the best of our knowledge, this is the first work to consider the interplay between these two realms of analysis. ${ }^{1}$

Our analysis reveals that as long the plant is stabilizable and detectable from all of its inputs and outputs taken together, and as long as the wireless network provides paths from certain plant sensors to certain other plant actuators, then for almost any choice of coefficients in the linear iterative strategy employed by the network nodes, a stabilizing compensator can be designed at each actuator. An interesting byproduct of our results is that stabilization is possible despite the length of the paths between the sensors and actuators, as long as compensators of sufficiently large order are allowed at the actuators. Furthermore, our scheme requires each node in the network to transmit its state only once per time-step of the plant, thereby maintaining the beneficial scheduling and compositionality aspects described in [5].

\section{NOTATION AND TERMINOLOGY}

We use $\mathbf{e}_{i}$ to denote the column vector (of appropriate size) with a 1 in its $i$-th position and 0's elsewhere. The symbol $\mathbf{I}_{N}$ denotes the $N \times N$ identity matrix, and $\mathbf{A}^{\prime}$ indicates the transpose of matrix $\mathbf{A}$. For a square matrix $\mathbf{M}, \Lambda(\mathbf{M})$ denotes the set of eigenvalues of $\mathbf{M}$. The cardinality of a set $\mathcal{S}$ is denoted by $|\mathcal{S}|$, and for two sets $\mathcal{S}$ and $\mathcal{R}$, we use $\mathcal{S} \backslash \mathcal{R}$ to denote the set of elements in $\mathcal{S}$ that are not in $\mathcal{R}$. Finally, we define the sets $\mathcal{M}=\{1,2, \ldots, m\}$ and $\mathcal{P}=\{1,2, \ldots, p\}$.

\section{A. Structured Linear Systems}

Consider a system $\Sigma$ of the form:

$$
\mathbf{x}[k+1]=\mathbf{A} \mathbf{x}[k]+\mathbf{B u}[k], \quad \mathbf{y}[k]=\mathbf{C x}[k],
$$

where $\mathbf{x}[k] \in \mathbb{R}^{n}, \mathbf{u}[k] \in \mathbb{R}^{m}, \mathbf{y}[k] \in \mathbb{R}^{p}$ and the matrices are of the appropriate dimensions. For convenience, we will denote the system as $\Sigma=(\mathbf{A}, \mathbf{B}, \mathbf{C})$.

A linear system of the form (1) is said to be structured if each entry in the system matrices is either a fixed zero or an independent free parameter [10]. A structured system $\Sigma$ can be represented via a directed graph $\mathcal{G}_{\Sigma}=\left\{\mathcal{V}_{\Sigma}, \mathcal{E}_{\Sigma}\right\}$. The vertex set is given by $\mathcal{V}_{\Sigma}=\{\mathcal{X} \cup \mathcal{U} \cup \mathcal{Y}\}$ where $\mathcal{X}=\left\{x_{1}, \ldots, x_{n}\right\}$ denotes the set of state vertices, while

\footnotetext{
${ }^{1}$ For instance, previous works that used graph-theory to analyze numerical systems were limited to the case where all eigenvalues had multiplicity equal to one (e.g., [9]), which is the generic case when the numerical parameters are abstracted away.

${ }^{2}$ We will sometimes refer to these graphs as structural graphs.
} 


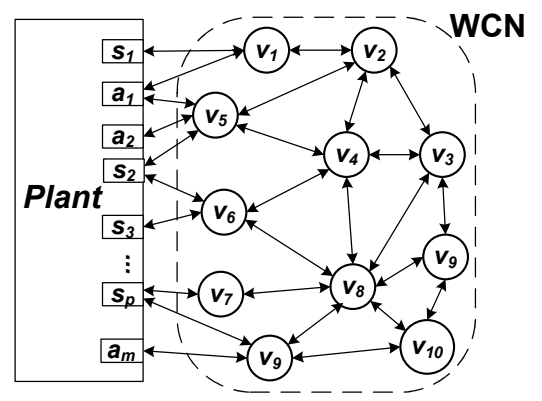

Fig. 1. A multi-hop WCN used as a distributed controller.

$\mathcal{U}=\left\{u_{1}, \ldots, u_{m}\right\}$ and $\mathcal{Y}=\left\{y_{1}, \ldots, y_{p}\right\}$ denote the sets of input and output vertices, respectively. The edge set is given by $\mathcal{E}_{\Sigma}=\mathcal{E}_{\mathbf{A}} \cup \mathcal{E}_{\mathbf{B}} \cup \mathcal{E}_{\mathbf{C}}$ with $\mathcal{E}_{\mathbf{A}}=\left\{\left(x_{i}, x_{j}\right) \mid a_{j i} \neq 0\right\}$, $\mathcal{E}_{\mathbf{B}}=\left\{\left(u_{i}, x_{j}\right) \mid b_{j i} \neq 0\right\}, \mathcal{E}_{\mathbf{C}}=\left\{\left(x_{i}, y_{j}\right) \mid c_{j i} \neq 0\right\}$. For a structured system, a simple path is called a $U$-rooted path if the path starts at a vertex in $\mathcal{U}$. A set of mutually disjoint U-rooted paths is called a $U$-rooted path family. Similarly, a simple path that ends at a vertex in $\mathcal{Y}$ is called a $Y$-topped path, while a set of mutually disjoint Y-topped paths is called a Y-topped path family.

We will be interested in properties of a structured system that can be inferred purely from the zero/nonzero structure of the system matrices. These properties will hold almost everywhere - for almost any choice of free parameters (i.e., the set of parameters for which the property does not hold has Lebesgue measure zero [10]). Two systems are called structurally equivalent if they have the same number of states, inputs and outputs, and their system matrices have zeros in the same locations.

\section{Wireless CONTROL NETWORK}

Consider the system in Fig. 1, where a wireless network is used to control a system $\Sigma=(\mathbf{A}, \mathbf{B}, \mathbf{C})$ with state $\mathbf{x} \in \mathbb{R}^{n}$, input $\mathbf{u} \in \mathbb{R}^{m}$ and output $\mathbf{y} \in \mathbb{R}^{p}$. The measurements of the output vector $\mathbf{y}[k]$ are provided by the sensors from the set $\mathcal{S}=\left\{s_{1}, s_{2}, \ldots, s_{p}\right\}$, while the input vector $\mathbf{u}[k]$ is applied to the plant by actuators from the set $\mathcal{A}=\left\{a_{1}, a_{2}, \ldots, a_{m}\right\}$.

The wireless network is described by a graph $\mathcal{G}=\{\mathcal{V}, \mathcal{E}\}$, where $\mathcal{V}=\left\{v_{1}, v_{2}, \ldots, v_{N}\right\}$ is the set of $N$ nodes and $\mathcal{E} \subseteq$ $\mathcal{V} \times \mathcal{V}$ represents the communication topology (i.e., edge $\left(v_{j}, v_{i}\right) \in \mathcal{E}$ if node $v_{i}$ can receive information directly from node $v_{j}$ ). We also define $\mathcal{V}_{S} \subset \mathcal{V}$ as the set of nodes that can receive information directly from at least one sensor, and $\mathcal{V}_{A} \subset \mathcal{V}$ as the set of nodes whose transmissions can be heard by at least one actuator. In addition, we define a new graph $\overline{\mathcal{G}}=\left\{\mathcal{V} \cup \mathcal{S} \cup \mathcal{A}, \mathcal{E} \cup \mathcal{E}_{\text {in }} \cup \mathcal{E}_{\text {out }}\right\}$ that includes the initial graph $\mathcal{G}$, the plant's sensors and actuators, and the edge sets:

$$
\begin{aligned}
& \mathcal{E}_{\text {out }}=\left\{\left(s_{l}, v_{i}\right) \mid \begin{array}{c}
s_{l} \in \mathcal{S}, v_{i} \in \mathcal{V}_{S}, \\
v_{i} \text { can receive values from sensor } s_{l}
\end{array}\right\}, \\
& \mathcal{E}_{\text {in }}=\left\{\left(v_{i}, a_{l}\right) \mid \begin{array}{c}
a_{l} \in \mathcal{A}, v_{i} \in \mathcal{V}_{A}, \\
\text { actuator } a_{l} \text { can receive values from } v_{i}
\end{array}\right\} .
\end{aligned}
$$

The WCN scheme proposed in [5] requires each wireless node to maintain a (possibly vector) state and to implement the following simple iterative procedure: at every time step (i.e., communication frame) each node in the network updates its state to be a linear combination of its previous state and its neighbors' states. Each node from the set $\mathcal{V}_{S}$ also includes a linear combination of the sensor measurements (i.e., plant outputs) from all sensors in its neighborhood. Denoting node $v_{i}$ 's state at time step $k$ by $z_{i}[k]$, the update procedure is given by: ${ }^{3}$

$$
z_{i}[k+1]=w_{i i} z_{i}[k]+\sum_{v_{j} \in \mathcal{N}_{v_{i}}} w_{i j} z_{j}[k]+\sum_{s_{j} \in \mathcal{N}_{v_{i}}} h_{i j} y_{j}[k] .
$$

For the scheme in [5], each plant input $u_{i}[k], i \in \mathcal{M}$ is taken to be a linear combination of values from the nodes in actuator $a_{i}$ 's neighborhood: ${ }^{4}$

$$
u_{i}[k]=\sum_{j \in \mathcal{N}_{a_{i}}} g_{i j} z_{j}[k] .
$$

Aggregating the state values of all nodes at time step $k$ into the value vector $\mathbf{z}[k]$, the state maintained by the network evolves according to the dynamics given by

$$
\begin{aligned}
\mathbf{z}[k+1] & =\mathbf{W} \mathbf{z}[k]+\mathbf{H y}[k], \\
\mathbf{u}[k] & =\mathbf{G z}[k]
\end{aligned}
$$

where $\mathbf{z}[k] \in \mathbb{R}^{N}$ if each node maintains a scalar state ${ }^{5}$ and matrices $\mathbf{W}, \mathbf{H}$ and $\mathbf{G}$ are of the appropriate dimensions. In the above equation, for all $i \in\{1, \ldots, N\}, w_{i j}=0$ if $v_{j} \notin \mathcal{N}_{v_{i}} \cup\left\{v_{i}\right\}, h_{i j}=0$ if $s_{j} \notin \mathcal{N}_{v_{i}}$, and $g_{i j}=0$ if $v_{j} \notin \mathcal{N}_{a_{i}}$. Therefore, the matrices $\mathbf{W}, \mathbf{H}$ and $\mathbf{G}$ are structured, with sparsity constraints determined by the WCN topology. Thus, the linear strategy employed by all nodes causes the entire network itself to behave as a structured dynamical compensator.

If the overall system state is denoted by $\hat{\mathbf{x}}[k]=$ $\left[\begin{array}{ll}\mathbf{x}[k]^{\prime} & \mathbf{z}[k]^{\prime}\end{array}\right]^{\prime}$, the closed-loop system can be described as:

$$
\hat{\mathbf{x}}[k+1]=\left[\begin{array}{cc}
\mathbf{A} & \mathbf{B G} \\
\mathbf{H C} & \mathbf{W}
\end{array}\right]\left[\begin{array}{l}
\mathbf{x}[k] \\
\mathbf{z}[k]
\end{array}\right] \triangleq \hat{\mathbf{A}} \hat{\mathbf{x}}[k] .
$$

Choosing $\mathbf{W}, \mathbf{G}$ and $\mathbf{H}$ to obtain a stable ${ }^{6} \hat{\mathbf{A}}$ can be cast in the form of a static output feedback problem with sparsity constraints on the gain matrix; this is a nonconvex problem, but various numerical procedures have been proposed in the literature (e.g., [13]). In [5], we adapted some of these numerical procedures to find values for the nonzero parameters in $\mathbf{W}, \mathbf{H}$ and $\mathbf{G}$ so that the matrix $\hat{\mathbf{A}}$ is stable, given a network topology and a predefined state size maintained by each node. However, the proposed procedure is iterative in nature, and convergence depends on the initialization point for the algorithm. Thus, even if a stabilizing configuration exists, the procedure might not be able to find it.

In this paper, we study topological conditions on the network that guarantee the existence of a stabilizing configuration. To facilitate our investigation, we focus on a WCN architecture where each wireless node maintains a scalar state, but where more computation can be assigned to the

\footnotetext{
${ }^{3}$ The neighborhood $\mathcal{N}_{v}$ of a vertex $v$ is with respect to the graph $\overline{\mathcal{G}}$.

${ }^{4} \mathrm{Eq}$. (5) captures the situation where the plant sensors and actuators are geographically separated, preventing the plant input from directly depending on any of the plant's outputs.

${ }^{5}$ In the general case $\mathbf{z}[k] \in \mathbb{R}^{N_{s}}$, where $N_{s}$ is a sum of state sizes of all nodes in the network. For more details see [5].

${ }^{6} \mathrm{We}$ refer to matrices $\mathbf{W}, \mathbf{H}$ and $\mathbf{G}$ that satisfy the topological constraints and guarantee stability of $\hat{\mathbf{A}}$ as a stabilizing configuration.
} 
actuators (essentially causing them to act as reduced order controllers). This scenario is motivated by practical reasons, since actuators are usually placed on 'fixed' positions and are not power constrained, allowing them to utilize more powerful CPUs. On the other hand, wireless nodes in the network are usually battery-operated low-power microcontrollers, which are not computationally powerful. To find conditions that guarantee stabilization in this case, we will make use of the concept of fixed modes in decentralized control systems.

\section{Decentralized Fixed Modes}

The decentralized fixed modes of a linear dynamical system are eigenvalues of the plant that cannot be moved by static output feedback, where the feedback gain matrix potentially has some sparsity constraints. The concept of fixed modes was initially introduced for decentralized continuoustime systems in [6] (where the gain matrix has a block diagonal structure), and was generalized in [12] to handle arbitrary feedback patterns, and to enable a graph-theoretic analysis of the problem. For the discrete-time case, the basic problem of system stabilization using decentralized feedback controllers (i.e., a problem equivalent to [6]) was considered in [14], where only algebraic conditions were derived.

Consider a discrete-time system $\Sigma=(\mathbf{A}, \mathbf{B}, \mathbf{C})$ controlled by a set of $m$ controllers where each controller is located at a different actuator, and has direct access to only a subset of the plant outputs.

Definition 1: The decentralized feedback structure constraints (i.e., patterns) are specified as $m$ sets $J_{1}, J_{2}, \ldots, J_{m} \subseteq$ $\mathcal{P}$ such that for each $i \in \mathcal{M}, j \in J_{i}$ if and only if output $y_{j}$ can be directly used to calculate input $u_{i}$.

Using the above definition, $m$ linear time-invariant dynamical feedback compensators are described as $(i=1, \ldots, m)$ :

$$
\begin{aligned}
\mathbf{z}_{i}[k+1] & =\mathbf{F}_{\mathbf{i}} \mathbf{z}_{i}[k]+\sum_{j \in J_{i}} \mathbf{q}_{i j} y_{j}[k] \\
u_{i}[k] & =\mathbf{h}_{i}^{\prime} \mathbf{z}_{i}[k]+\sum_{j \in J_{i}} k_{i j} y_{j}[k],
\end{aligned}
$$

where $\mathbf{z}_{i} \in \mathbb{R}^{n_{i}}$ is the controller's state vector (of size determined by the nature of the plant and the feedback patterns), while matrix $\mathbf{F}_{i}$ and vectors $\mathbf{q}_{i}, \mathbf{h}_{i}$ are of the appropriate dimensions. Based on the feedback patterns $J_{1}, J_{2}, \ldots, J_{m}$, define the set $\mathbf{K}_{f}=\left\{\mathbf{K} \in \mathbb{R}^{m \times p} \mid k_{i j}=0\right.$ if $\left.j \notin J_{i}\right\}$.

Definition 2 ([6], [12]): For the system $\Sigma=(\mathbf{A}, \mathbf{B}, \mathbf{C})$, the set $\Lambda_{f}=\bigcap_{\mathbf{K} \in \mathbf{K}_{f}} \Lambda(\mathbf{A}+\mathbf{B K} \mathbf{C})$ is called the set of fixed modes with respect to the feedback structure constraints specified by $J_{1}, J_{2}, \ldots, J_{m}$.

The following classical result explains the vital role that fixed modes play in the stabilizability analysis of linear dynamical systems.

Theorem 1 ([6]): The system $\Sigma$ can be stabilized using the set of controllers defined in (9) if and only if all fixed modes are stable.

For any subset $I \subseteq \mathcal{M}$ we define $J=\bigcup_{i \in \mathcal{M} \backslash I} J_{i}$. The following theorem characterizes the fixed modes of a given system with respect to the feedback pattern $J_{1}, J_{2}, \ldots, J_{m}$.
Theorem 2 ([8]): A complex number $\lambda$ is a fixed mode of the system $\Sigma=(\mathbf{A}, \mathbf{B}, \mathbf{C})$ if and only if there exists a subset $I \subseteq \mathcal{M}$ such that

$$
\operatorname{rank}\left[\begin{array}{cc}
\mathbf{A}-\lambda \mathbf{I} & \mathbf{B}_{I} \\
\mathbf{C}_{J} & \mathbf{0}
\end{array}\right]<n,
$$

where $\mathbf{B}_{I}$ and $\mathbf{C}_{J}$ are the columns and rows of $\mathbf{B}$ and $\mathbf{C}$ indexed by the elements in sets $I$ and $J$, respectively.

As described in [7], there are two distinct sources for a fixed mode. A fixed mode can either arise from a loss of rank due to a 'perfect cancellation' of the numerical parameters (which is a degenerate case), or it can be caused by deeper issues relating to the system structure. The later type of fixed modes are called structural fixed modes.

Definition 3 ([7]): The system $\Sigma$ has structural fixed modes with respect to the feedback constraints from (9) if every system structurally equivalent to $\Sigma$ has fixed modes with the same feedback constraints.

As described in Section II-A, one can associate a graph $\mathcal{G}_{\Sigma}=\left\{\mathcal{V}_{\Sigma}, \mathcal{E}_{\Sigma}\right\}$ with a given system $\Sigma$. The graph can be augmented to capture a given feedback pattern $J_{1}, J_{2} \ldots, J_{m}$ via a set of edges $\mathcal{E}_{J}=\left\{\left(y_{j}, u_{i}\right) \mid i \in \mathcal{M}, j \in J_{i}\right\}$. This produces the graph $\mathcal{G}_{\Sigma, J}=\left\{\mathcal{V}_{\Sigma}, \mathcal{E}_{\Sigma} \cup \mathcal{E}_{J}\right\}$. From the graph $\mathcal{G}_{\Sigma, J}$, using a similar approach to [12], we can state the following result that specifies a graph-theoretic characterization for structural fixed modes (for more details see [15]).

Lemma 1: When a feedback structure $J_{1}, \ldots, J_{m}$ is used, $\Sigma$ has no structural fixed modes (other than at the origin) if and only if each state vertex $x_{k} \in \mathcal{X}$ is contained in a strong component of $\mathcal{G}_{\Sigma, J}$ that includes an edge from $\mathcal{E}_{J}$.

The above lemma does not preclude structural fixed modes at zero. ${ }^{7}$ Although such modes are a concern for continuoustime systems, they are not an issue for stabilization of discrete-time plants. Thus, the lemma specifies sufficient conditions for the existence of a set of stabilizing feedback controllers for almost every plant that has the given structure, with the given feedback pattern. Two caveats are in order. First, the theorem does not specify the size of the stabilizing controllers (i.e., the values for $n_{i}, i=1, \ldots, m$ from (9)); only that sufficiently large controllers can be found for each actuator to jointly stabilize the system. The second caveat is that the existing analysis of decentralized feedback control systems assumes that each actuator has direct access to at least one of the plant outputs (i.e., the quantities $\mathbf{q}_{i j}$ and $k_{i j}$ in (9) are nonzero). This leads to a nonempty set $\mathbf{K}_{f}$ and this assumption is utilized in the proof of sufficiency from [6] to show that all non-fixed modes can be stabilized.

These caveats prevent Lemma 1 from being directly used to analyze whether the system can be stabilized using a WCN. First, we would like the wireless nodes to maintain only small state vectors (ideally scalars). Second, each actuator generally only has access to the transmissions of nearby wireless nodes, and not the plant outputs directly (thus $\mathbf{K}_{f}$ contains only the zero matrix). Therefore, in this case, the role of fixed modes in stabilization over a network must be carefully studied. This is the objective of the rest of the paper.

\footnotetext{
${ }^{7}$ It can be shown that the system does not have structured fixed modes at the origin if and only if there exist a set of disjoint cycles in the graph $\mathcal{G}_{\Sigma, J}$ that covers all state vertices.
} 
To illustrate the limitations of the conditions from Lemma 1 when a WCN is used, consider the example from Fig. 2(a) where the plant described by the matrices

$$
\mathbf{A}=\left[\begin{array}{ccc}
1 & 1 & 0 \\
0 & 1 & 1 \\
0 & 0 & 2.1
\end{array}\right], \quad \mathbf{B}=\left[\begin{array}{c}
0 \\
0.5 \\
1
\end{array}\right], \quad \mathbf{C}=\left[\begin{array}{lll}
1 & 0.5 & 1
\end{array}\right],
$$

is to be controlled using a WCN consisted of two nodes. The graph $\mathcal{G}_{\Sigma, J}$ of the system is presented in Fig. 2(b). In this case, the WCN, which acts a dynamic compensator, has access to all of the plant outputs and inputs (i.e., $J_{1}=$ $\mathcal{P}=\{1\}$ ), and the conditions from Lemma 1 are satisfied. However, since the WCN is a structured controller (due to its sparsity constraints), it can be shown that in this case there is no stabilizing configuration for the WCN when each node maintains a scalar state. ${ }^{8}$ Thus, the necessary and sufficient conditions from Lemma 1 do not appear to hold in this case; later, we will show how to address this by allowing the actuator to maintain a larger state, while the other nodes maintain scalar states.

\section{Structural Fixed Modes With a Wireless CONTROL NETWORK}

In this section, we provide conditions for a given system to not have structural fixed modes when controlled over a WCN, where each node in the network maintains only a scalar state, the actuator nodes maintain vector states, and no actuator has direct access to any plant output.

Consider the plant $\Sigma=(\mathbf{A}, \mathbf{B}, \mathbf{C})$ and the WCN together as a linear system $\tilde{\Sigma}$, where the outputs of the plant are injected into the WCN. At each time-step the plant actuators receive the transmissions from the wireless nodes in the set $\mathcal{V}_{A}$, but suppose for now that the plant actuators do not use these transmissions to close the loop. If we view the transmissions of the nodes in $\mathcal{V}_{A}$ as the output of the system $\tilde{\Sigma}$, the system can be specified as: ${ }^{9}$

$$
\begin{gathered}
\hat{\mathbf{x}}[k+1]=\left[\begin{array}{l}
\mathbf{x}[k+1] \\
\mathbf{z}[k+1]
\end{array}\right]=\underbrace{\left[\begin{array}{cc}
\mathbf{A} & \mathbf{0} \\
\mathbf{H C} & \mathbf{W}
\end{array}\right]}_{\tilde{\mathbf{A}}}\left[\begin{array}{c}
\mathbf{x}[k] \\
\mathbf{z}[k]
\end{array}\right]+\underbrace{\left[\begin{array}{c}
\mathbf{B} \\
\mathbf{0}
\end{array}\right]}_{\tilde{\mathbf{B}}} \mathbf{u}[k], \\
\hat{\mathbf{y}}[k]=\underbrace{\left[\begin{array}{ll}
\mathbf{0} & \mathbf{E}_{\mathcal{V}_{A}}
\end{array}\right]}_{\tilde{\mathbf{C}}}\left[\begin{array}{c}
\mathbf{x}[k]] \\
\mathbf{z}[k]
\end{array}\right],
\end{gathered}
$$

with $\mathbf{E}_{\mathcal{V}_{A}}=\left[\begin{array}{llll}\mathbf{e}_{i_{1}} & \mathbf{e}_{i_{2}} & \ldots & \mathbf{e}_{i_{t}}\end{array}\right]^{\prime}$ selecting the state values from the set $\mathcal{V}_{A}=\left\{v_{i_{1}}, v_{i_{2}}, \ldots, v_{i_{t}}\right\}$ (where $t=\left|\mathcal{V}_{A}\right|$ ).

The structural graph $\mathcal{G}_{\tilde{\Sigma}}=\left\{\mathcal{V}_{\tilde{\Sigma}}, \mathcal{E}_{\tilde{\Sigma}}\right\}$ of the system $\tilde{\Sigma}$ is obtained by composing the structural graph of the initial plant $\Sigma$ and the WCN graph $\mathcal{G}=\{\mathcal{V}, \mathcal{E}\}$, where $\mathcal{V}_{\tilde{\Sigma}}=\mathcal{X} \cup \mathcal{U} \cup \mathcal{V}$, $\mathcal{E}_{\tilde{\Sigma}}=\mathcal{E}_{\mathbf{A}} \cup \mathcal{E} \cup \mathcal{E}_{\tilde{\mathcal{O}}}$ and (for $\mathcal{E}_{\text {out }}$ defined in (2))

$$
\mathcal{E}_{\tilde{\mathcal{O}}}=\left\{\left(x_{i}, v_{j}\right) \in \mathcal{X} \times \mathcal{V}_{S} \mid \exists y_{k},\left(x_{i}, y_{k}\right) \in \mathcal{E}_{\mathbf{C}},\left(y_{k}, v_{j}\right) \in \mathcal{E}_{\text {out }}\right\}
$$

\footnotetext{
${ }^{8}$ To verify that this was caused by structural fixed modes, we have tried to stabilize several plants with the same structure and different parameters. However, in all the cases a stabilizing configuration could not be extracted.

${ }^{9}$ For technical reasons, we assume that the matrices $\mathbf{H}$ and $\mathbf{C}$ satisfy the property that either $\mathbf{H}$ has a single nonzero entry in each column, or $\mathbf{C}$ has a single nonzero entry in each row. This assumption guarantees that each nonzero entry in the product $\mathbf{H C}$ will be an independent free parameter, if each nonzero entry in $\mathbf{H}$ and $\mathbf{C}$ is treated as independent free parameter. This assumption can be satisfied by having a dedicated wireless node for each plant output.
}
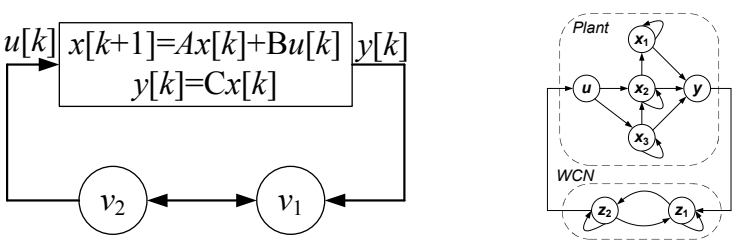

Fig. 2. (a) An example of a WCN; (b) Graph description of the system.

is the edge set between the state vertices connected to a plant output and all network nodes in the neighborhood of the corresponding plant sensor. ${ }^{10}$

In the basic WCN scheme each actuator applies a scalar linear combination of its neighboring nodes' states (i.e., see (5)). However, suppose that we allow each actuator $a_{i},(1 \leq i \leq m)$ to maintain a possible vector state denoted by $\mathbf{z}_{a_{i}} \in \mathbb{R}^{n_{i}}$. The procedure implemented by the actuator can be described as: ${ }^{11}$

$$
\begin{aligned}
\mathbf{z}_{a_{i}}[k+1] & =\mathbf{W}_{a_{i}} \mathbf{z}_{a_{i}}[k]+\sum_{v_{j} \in \mathcal{N}_{a_{i}}} \mathbf{g}_{i j} z_{j}[k] \\
u_{i}[k] & =\mathbf{t}_{a_{i}}^{\prime} \mathbf{z}_{a_{i}}[k]+\sum_{v_{j} \in \mathcal{N}_{a_{i}}} k_{i j} z_{j}[k],
\end{aligned}
$$

for some matrices $\mathbf{W}_{a_{i}}$, vectors $\mathbf{g}_{i j}, \mathbf{t}_{a_{i}}$ and scalars $k_{i j}$.

In this setup, the overall system $\Sigma$ in (12) is to be controlled with a set of $m$ decentralized feedback controllers described by (13). In addition, the feedback pattern is specified with the edge set $\mathcal{E}_{i n}$ from (3) (i.e., in this case $\left.\mathcal{E}_{J}=\mathcal{E}_{i n}\right)$. The key insight is the following: by having each wireless node run a linear strategy, the WCN and the plant together form a linear system $\tilde{\Sigma}$. Then, by viewing the transmissions of the wireless nodes closest to the actuators as the new 'outputs' of the system $\tilde{\Sigma}$, the problem of stabilizing the system with compensators at the actuators fits within the classical decentralized control formulation described in Section IV (where each input has direct access to some outputs). Therefore, with this insight, we can apply Lemma 1 to obtain the following topological condition that guarantees the existence of a stabilizing configuration.

Theorem 3: Consider system $\Sigma=(\mathbf{A}, \mathbf{B}, \mathbf{C})$ and a WCN where each actuator acts as a dynamical compensator. For each plant state vertex $x_{i} \in \mathcal{X}$ in the structural graph $\mathcal{G}_{\Sigma}$, let $A_{i}$ denote the set of input vertices from which $x_{i}$ is reachable in the initial system, while $\mathcal{V}_{A_{i}}$ denotes the set of the WCN nodes that are neighbors of the actuators in $A_{i}$. Then, almost any system structurally equivalent to $\Sigma$ can be stabilized with the WCN if in the corresponding structural graph $\mathcal{G}_{\tilde{\Sigma}}=\left\{\mathcal{V}_{\tilde{\Sigma}}, \mathcal{E}_{\tilde{\Sigma}}\right\}$ every plant state vertex $x_{i}$ has a path to a WCN state vertex from $\mathcal{V}_{A_{i}}$.

Proof: Consider the graph $\mathcal{G}_{\tilde{\Sigma}}=\left\{\mathcal{V}_{\tilde{\Sigma}}, \mathcal{E}_{\tilde{\Sigma}}\right\}$ of the structured system (12) composed of the plant and the WCN. If for a plant state vertex $x_{i}$ there exists a WCN state vertex

\footnotetext{
${ }^{10}$ These edges appear as a result of the product HC. Since all nonzero entries of $\mathbf{H C}$ are independent, the set of plant output vertices $\mathbf{Y}$ do not appear in the graph, and the wireless node vertices are directly connected to the plant state vertices.

${ }^{11}$ For each actuator $a_{i}$ and each node $v_{j} \in \mathcal{N}_{a_{i}}$ there exists some row $l$ of $\hat{\mathbf{y}}[k]$ in (12) such that $z_{j}[k]=\hat{y}_{l}[k]$. Therefore, the terms $\sum_{v_{j} \in \mathcal{N}_{a_{i}}} \mathbf{g}_{i j} z_{j}[k]$ and $\sum_{v_{j} \in \mathcal{N}_{a_{i}}} k_{i j} z_{j}[k]$ correspond to linear combinations of the WCN outputs $\hat{\mathbf{y}}[k]$.
} 
$z_{j} \in \mathcal{V}_{A_{i}}$ reachable from $x_{i}$, then $x_{i}$ belongs to a strong component with an edge from $\mathcal{E}_{i n}$. Since this holds for all state vertices, Lemma 1 is satisfied for these vertices.

On the other hand, a fixed mode will be introduced with each WCN state vertex $z_{i}$ that does not belong to a strong component in the graph $\mathcal{G}_{\tilde{\Sigma}_{i n}}=\left\{\mathcal{V}_{\tilde{\Sigma}}, \mathcal{E}_{\tilde{\Sigma}} \cup \mathcal{E}_{i n}\right\}$ with an edge from $\mathcal{E}_{\text {in }}$ (this might happen if the network is disconnected). However, by setting to zero all the weights associated with the links outgoing from $z_{i}$ it is ensured that this WCN state vertex is effectively removed from the network. In this case, due to the state vertex $z_{i}$ the system will have an additional structured fixed mode in the origin. Thus, in both cases the closed-loop system does not have structured fixed-modes outside of zero, meaning that almost every system with this structure will be stabilizable using the WCN.

If the condition in the above theorem is satisfied, a stabilizing configuration for the WCN with dynamical compensators can be found via a simple modification of the numerical procedure for the basic WCN described in [5] (for more details see [15]). For example, we have found that the system from Fig. 2 and (11) satisfies the conditions in the above theorem, and using the procedure from [15], it can be stabilized with the WCN if each node maintains a scalar state and the actuator maintains a state from $\mathbb{R}^{2}$.

\section{Minimal Stabilizing Feedback Connections}

The previous section describes conditions that guarantee that a system does not have structural fixed modes when controlled over a WCN. In this section, we investigate the minimal connectivity that the WCN should provide to ensure that the conditions from the previous section hold.

For decentralized continuous-time systems, [16] considered the problem of determining the minimal number of direct connections between plant outputs and inputs to ensure that the system does not have structured fixed modes. Leveraging the fact that fixed modes at zero do not cause problems for the stabilization of discrete-time systems, we now present a simplified procedure that can be used to determine a minimal set of feedback edges that guarantee the absence of nonzero structural fixed modes (again, when direct plant output-input connections are allowed). We will then use this to infer properties that the WCN should satisfy in order to stabilize the plant.

Consider a system $\Sigma=(\mathbf{A}, \mathbf{B}, \mathbf{C})$. For all sets $I \subseteq \mathcal{M}$ and $J \subseteq \mathcal{P}$ we denote with $\mathbf{B}_{I}$ and $\mathbf{C}_{J}$ submatrices of $\mathbf{B}$ and $\mathbf{C}$ consisting of columns of $\mathbf{B}$ and rows of $\mathbf{C}$ with indices in $I$ and $J$, respectively. A system $\Sigma_{I J}=\left(\mathbf{A}, \mathbf{B}_{I}, \mathbf{C}_{J}\right)$ can be described with a graph $\mathcal{G}_{\Sigma_{I J}}=\left\{\mathcal{V}_{\Sigma_{I J}}, \mathcal{E}_{\Sigma_{I J}}\right\}$, which can be obtained from $\mathcal{G}_{\Sigma}=\left\{\mathcal{V}_{\Sigma}, \mathcal{E}_{\Sigma}\right\}$ by keeping input vertices from the index set $I$ and output vertices associated with set $J$. These sets are denoted $\mathcal{U}_{I}$ and $\mathcal{Y}_{J}$, respectively. Since we consider structurally controllable and observable systems, we will use the following results that specify a set of conditions for structural controllability/observability.

Theorem 4 ([10]): A structured system is structurally controllable (observable) if and only if each state vertex is the end (beginning) of a U-rooted (Y-topped) path and there exists a disjoint union of a U-rooted (Y-topped) path family and a cycle family that covers all state vertices.
As with the case of fixed modes, uncontrollable and unobservable modes at the origin are not a major concern for discrete-time systems, and thus we present the following simplified tests for structural stabilizability and detectability, rather than observability and controllability.

Corollary 1: A structured system is structurally stabilizable (detectable) if each state vertex is the end (beginning) of a U-rooted (Y-topped) path.

Definition 4: A stabilizable subset of the plant inputs is a set $I \subseteq \mathcal{M}$ such that $\left(\mathbf{A}, \mathbf{B}_{I}\right)$ is structurally stabilizable. Similarly, a detectable subset of the outputs is a set $J \subseteq \mathcal{P}$ for which $\left(\mathbf{A}, \mathbf{C}_{J}\right)$ is structurally detectable.

For some stabilizable subsets $I$, it may be possible to find an even smaller stabilizable subset $I^{\prime} \subset I$. Since we wish to investigate the minimal feedback requirements, we define the notion of essential input and output sets.

Definition 5: A stabilizable subset $I$ is called an essential input set if there is no structurally stabilizable (strict) subset $I^{\prime} \subset I$. Similarly, a detectable subset $J$ is called an essential output set if there is no structurally detectable (strict) subset $J^{\prime} \subset J$.

Note that for a particular system $\Sigma=(\mathbf{A}, \mathbf{B}, \mathbf{C})$ there might exist several different essential input and output sets. In addition, these sets could have different numbers of elements. We use the essential input and output sets to determine the minimal number of feedback connections that would guarantee that a system does not have nonzero structural fixed modes. From Lemma 1, for essential input and output sets $I$ and $O$, at least $\max (|I|,|O|)$ feedback connections have to be used. We use the theorem below to show that this number of feedback connections is also sufficient.

Theorem 5: For a structurally stabilizable and detectable system $\Sigma=(\mathbf{A}, \mathbf{B}, \mathbf{C})$, let $I$ and $O$ be essential input and outputs sets, respectively. Then the system can be stabilized by introducing $\max (|I|,|O|)$ feedback connections (directly between certain appropriately chosen outputs and inputs). $\square$

We will provide a sketch of the proof here. For the full proof, see [15].

Proof: A directed graph $\mathcal{G}_{\Sigma}=\left\{\mathcal{V}_{\Sigma}, \mathcal{E}_{\Sigma}\right\}$, representing the structured system $\Sigma$ can be uniquely decomposed into $k$ strongly connected components $\mathcal{C}=\left\{\mathcal{C}_{1}, \ldots, \mathcal{C}_{k}\right\}$. A component $\mathcal{C}_{i}$ is referred to as a root component if no vertex in the component has incoming edges from vertices in any other component. Furthermore, $\mathcal{C}_{j}$ is called a leaf component if no vertex in $\mathcal{C}_{j}$ has an outgoing edge to a vertex in any other component.

Consider a directed acyclic graph $\mathcal{G}_{\mathcal{C}}=\left\{\mathcal{C} \cup \mathcal{U}_{I} \cup \mathcal{Y}_{J}, \mathcal{E}_{\mathcal{C}} \cup\right.$ $\left.\mathcal{E}_{\mathcal{I C}} \cup \mathcal{E}_{\mathcal{J C}}\right\}$, where $\left(\mathcal{C}_{i}, \mathcal{C}_{j}\right) \in \mathcal{E}_{\mathcal{C}}$ if and only if component $\mathcal{C}_{j}$ has an incoming edge from a vertex in $\mathcal{C}_{i}$, and

$$
\begin{gathered}
\mathcal{E}_{\mathcal{I C}}=\left\{\begin{array}{l|c}
\left(u_{i}, \mathcal{C}_{t}\right) \mid \begin{array}{c}
i \in I, \mathcal{C}_{t} \text { is a root component from } \mathcal{C}, \\
\mathcal{C}_{t} \text { has an edge from input vertex } u_{i}
\end{array}
\end{array}\right\}, \\
\mathcal{E}_{C O}=\left\{\begin{array}{l|c}
j \in J, \mathcal{C}_{t} \text { is a leaf component in } \mathcal{C}, \\
\text { output vertex } \left.y_{j} \text { has an edge from } \mathcal{C}_{t}, y_{j}\right)
\end{array}\right\} .
\end{gathered}
$$

Since the system $\Sigma$ is structurally stabilizable and detectable each leaf component has to be connected to an output vertex $y_{j} \in \mathcal{Y}_{J}$ and each root component is connected to an input vertex $u_{i} \in \mathcal{U}_{I}$.

We now use the following algorithm to introduce a set of feedback links between output vertices from $\mathcal{Y}_{J}$ and 
input vertices from $\mathcal{U}_{I}$. This set, denoted $\mathcal{E}_{\mathcal{F}}$, contains $\max (|I|,|O|)$ links and satisfies the conditions from Lemma 1 (for details see [15]). Thus, a feedback pattern consisting only of $\max (|I|,|O|)$ is sufficient to ensure that the system has no nonzero structurally fixed modes.

\begin{tabular}{l}
\hline Algorithm 1 Creating a minimal set of feedback connections \\
\hline 1. Select an input vertex $u_{i_{1}} \in \mathcal{U}_{I}$ and a corresponding \\
output vertex $y_{j_{1}} \in \mathcal{Y}_{J}$ such that $y_{j_{1}}$ is reachable from \\
$u_{i_{1}}$ in the graph $\mathcal{G}_{\mathcal{C}}$.
\end{tabular}

2. At iteration $t \geq 1$, select an input vertex $u_{i_{t+1}} \in$ $\mathcal{U}_{I} \backslash\left\{u_{i_{1}}, \ldots, u_{i_{t}}\right\}$ such that there exists an output vertex $y_{j_{t+1}} \in \mathcal{Y}_{J} \backslash\left\{y_{j_{1}}, \ldots, y_{j_{t}}\right\}$ reachable from $u_{i_{t+1}}$ in the initial graph $\mathcal{G}_{\mathcal{C}}$. If such an input $u_{i_{t+1}}$ does not exist, add the edge $\left(y_{j_{t}}, u_{i_{1}}\right)$ to $\mathcal{E}_{\mathcal{F}}$, and go to the next step. Otherwise, add the edge $\left(y_{j_{t}}, u_{i_{t+1}}\right)$ to the set $\mathcal{E}_{\mathcal{F}}$, set $t \leftarrow t+1$ and repeat step 2 .

3. If $\left\{u_{i_{1}}, \ldots, u_{i_{t}}\right\} \neq I$ and $\left\{y_{j_{1}}, \ldots, y_{j_{t}}\right\} \neq J$ then select $u_{i_{t+1}} \notin\left\{u_{i_{1}}, \ldots, u_{i_{t}}\right\}$ and $y_{j_{t+1}} \notin\left\{y_{j_{1}}, \ldots, y_{j_{t}}\right\}$ and add the edge $\left(y_{j_{t+1}}, u_{i_{t+1}}\right)$ to $\mathcal{E}_{\mathcal{F}}$. Set $t \leftarrow t+1$ and repeat step 3.

4. If $\left\{u_{i_{1}}, \ldots, u_{i_{t}}\right\}=I$ then for all $y_{j} \notin\left\{y_{j_{1}}, \ldots, y_{j_{t}}\right\}$ add the edge $\left(y_{j}, u_{i}\right)$ to $\mathcal{E}_{\mathcal{F}}$, where $u_{i}$ is an input vertex from which $y_{j}$ can be reached in the initial graph $\mathcal{G}_{\mathcal{C}}$.

5. If $\left\{y_{j_{1}}, \ldots, y_{j_{t}}\right\}=J$ then for all $u_{i} \notin\left\{u_{i_{1}}, \ldots, u_{i_{t}}\right\}$ add the edge $\left(y_{j}, u_{i}\right)$ to $\mathcal{E}_{\mathcal{F}}$, where $y_{j}$ is an output vertex reachable from $u_{i}$ in the initial graph $\mathcal{G}_{\mathcal{C}}$.

We now apply the above result to the case where a WCN is used for control (for situations where direct edges between plant inputs and outputs cannot be introduced). As mentioned earlier, the key trick is to view the composition of the WCN and the plant as a new dynamical system. Here, the set of nodes $\mathcal{V}_{\mathcal{A}}$ (in the neighborhood of the actuators) corresponds to the outputs of the new system. The new system will be structurally detectable if there exists a path between each essential plant output and a node from $\mathcal{V}_{\mathcal{A}}$. Therefore, we introduce the following results.

Definition 6: A detectable set of WCN nodes $\mathcal{V}_{\mathcal{D E} \mathcal{T}} \subseteq \mathcal{V}_{\mathcal{A}}$ is a set of nodes such that for each sensor $s_{j}$ that corresponds to an output $y_{j}$ from an essential output set $J$, there exists a path from $s_{j}$ to a node from $\mathcal{V}_{\mathcal{D E} \mathcal{T}}$.

Corollary 2: Consider a structurally stabilizable and detectable system $\Sigma(\mathbf{A}, \mathbf{B}, \mathbf{C})$ with essential input and output sets $I$ and $O$. The system can be stabilized with a WCN described by a graph $\mathcal{G}=\{\mathcal{V}, \mathcal{E}\}$ using $\max \left(|I|,\left|\mathcal{V}_{\mathcal{D E} \mathcal{T}}\right|\right)$ links between the nodes from a detectable set $\mathcal{V}_{\mathcal{D E T}}$ and actuators corresponding to the essential input set $I$.

The proof of the above corollary is readily obtained by noticing that if such a detectable set of nodes $\mathcal{V}_{\mathcal{D E T}} \subseteq \mathcal{V}_{\mathcal{A}}$ exists, then due to structural detectability of the plant there would be a path between each plant state vertex and a vertex representing the state of a node from $\mathcal{V}_{\mathcal{D E} \mathcal{T}}$. In addition, all network nodes that do not have a path to at least one node from $\mathcal{V}_{\mathcal{D E} \mathcal{T}}$ can be disregarded as in the proof of Theorem 3 (by setting all weights related to them to be zero). Thus, the 'new' system $\tilde{\Sigma}$ that contains the plant and the network is structurally detectable. Similarly, it can be shown that the 'new' system is stabilizable and the proof follows from Theorem 5, and by applying Algorithm 1.

Note, however, that there is a possibility that the feedback edges created by Algorithm 1 cannot be physically implemented, because it might cause an actuator to rely on a wireless node that is not actually in its neighborhood. The following corollary introduces a straightforward condition to preclude this case, and for designing WCNs that guarantee stabilization of almost all systems having a certain structure.

Corollary 3: Almost every structurally stabilizable and detectable system $\Sigma$ can be stabilized if the following conditions are met:

i. The WCN is strongly connected.

ii. There exists an essential output set, where each sensor in the set is connected to the network.

iii. There exists an essential input set, where each actuator in the set is connected to the network.

\section{Designing THE WCN TOPOLOGY TO STABILIZE A NUMERICALLY SPECIFIED PLANT}

In the previous sections, we have been focused on designing a WCN for a plant from a purely structural perspective; in other words, we considered only the interconnections between the plant state variables, but did not consider the numerical values of those interconnections. This allowed us to characterize WCN properties that would guarantee stabilization of almost any plant having a certain structure. However, one may be interested in designing a WCN for a given (numerically specified) system $\Sigma=(\mathbf{A}, \mathbf{B}, \mathbf{C})$. If this system falls within the measure zero set that is not covered by the structural analysis, one has to be more careful in designing the WCN. Specifically, any plant that has eigenvalues of multiplicity larger than 1 will not be captured by the generic set [8], and we will show that the multiplicity of eigenvalues in the plant will require the $\mathrm{WCN}$ to contain linkings of a sufficiently large size. ${ }^{12}$

Consider a WCN used to control a given (numerically specified) system $\Sigma=(\mathbf{A}, \mathbf{B}, \mathbf{C})$, where the pair $(\mathbf{A}, \mathbf{C})$ is detectable, and the pair $(\mathbf{A}, \mathbf{B})$ is stabilizable. Assuming for now that the plant actuators do not close the loop via the transmissions of nearby wireless nodes, the overall system $\tilde{\Sigma}=(\tilde{\mathbf{A}}, \tilde{\mathbf{B}}, \tilde{\mathbf{C}})$ (plant and wireless network) is given by (12). As in the previous sections, we consider the following problem. How should the WCN be designed to guarantee that a dynamic compensator can be designed at each actuator to stabilize the system, when each actuator only receives information about the output of the system via the WCN (and not directly)?

To answer this, for any actuator $a_{i}, i \in \mathcal{M}$, let $\mathcal{V}_{a_{i}}$ denote the WCN nodes whose transmissions can be heard by $a_{i}$. For any set $I \subseteq \mathcal{M}$, define $\mathcal{V}_{\mathcal{M} \backslash I}=\bigcup_{i \in \mathcal{M} \backslash I} \mathcal{V}_{a_{i}}$. In words, $\mathcal{V}_{\mathcal{M} \backslash I}$ is the set of all WCN nodes that are in

\footnotetext{
${ }^{12}$ For a directed graph $\mathcal{G}=\{\mathcal{V}, \mathcal{E}\}$, given two subsets $\mathcal{V}_{1}, \mathcal{V}_{2} \subset \mathcal{V}$, an $r$-linking from $\mathcal{V}_{1}$ to $\mathcal{V}_{2}$ is a set of $r$ vertex disjoint paths, each with start vertex in $\mathcal{V}_{1}$ and end vertex in $\mathcal{V}_{2}$.
} 
the neighborhood of actuators not in $I$. To show that the system (12) has no fixed modes with respect to the feedback structure $\mathcal{V}_{a_{1}}, \ldots, \mathcal{V}_{a_{m}}$, we will use Theorem 2 to show that

$$
\operatorname{rank} \underbrace{\left[\begin{array}{ccc}
\mathbf{A}-\lambda \mathbf{I} & \mathbf{0} & \mathbf{B}_{I} \\
\mathbf{H C} & \mathbf{W}-\lambda \mathbf{I} & \mathbf{0} \\
\mathbf{0} & \mathbf{E}_{F} & \mathbf{0}
\end{array}\right]}_{\tilde{\mathbf{M}}_{I, F}(\lambda)} \geq n+N
$$

for all unstable eigenvalues $\lambda$ of the matrices $\mathbf{A}$ or $\mathbf{W}$. Here, $\mathbf{E}_{F}$ is a matrix with a single 1 in each row, selecting the portions of the WCN state vector $\mathbf{z}[k]$ corresponding to the nodes in $\mathcal{V}_{\mathcal{M} \backslash I}$.

First, consider an unstable eigenvalue $\lambda$ of $\mathbf{A}$. Assume that $\lambda$ is not an eigenvalue of $\mathbf{W} .{ }^{13}$ Then, for any $I \subseteq \mathcal{M}$, the matrix $\tilde{\mathbf{M}}_{I, F}$ from (14) has rank

$$
\begin{aligned}
\operatorname{rank}\left(\tilde{\mathbf{M}}_{I, F}(\lambda)\right) & =\operatorname{rank}\left[\begin{array}{ccc}
\mathbf{A}-\lambda \mathbf{I} & \mathbf{0} & \mathbf{B}_{I} \\
\mathbf{0} & \mathbf{W}-\lambda \mathbf{I} & \mathbf{0} \\
\mathbf{E}_{F}(\mathbf{W}-\lambda \mathbf{I})^{-1} \mathbf{H C} & \mathbf{0} & \mathbf{0}
\end{array}\right] \\
& =N+\operatorname{rank}\left[\begin{array}{cc}
\mathbf{A}-\lambda \mathbf{I} & \mathbf{B}_{I} \\
\mathbf{E}_{F}(\mathbf{W}-\lambda \mathbf{I})^{-1} \mathbf{H C} & \mathbf{0}
\end{array}\right] .
\end{aligned}
$$

Thus, $\lambda$ is a fixed mode of $\tilde{\Sigma}=(\tilde{\mathbf{A}}, \tilde{\mathbf{B}}, \tilde{\mathbf{C}})$ with respect to $\mathcal{V}_{a_{1}}, \mathcal{V}_{a_{2}}, \ldots, \mathcal{V}_{a_{m}}$ if and only if it is a fixed mode of $\left(\mathbf{A}, \mathbf{B}, \mathbf{E}_{F}(\mathbf{W}-\lambda \mathbf{I})^{-1} \mathbf{H C}\right)$, with respect to the feedback pattern $\mathcal{V}_{a_{1}}, \mathcal{V}_{a_{2}}, \ldots, \mathcal{V}_{a_{m}}$. For any set $I \subseteq \mathcal{M}$, let

$$
\operatorname{rank}\left[\begin{array}{cc}
\mathbf{A}-\lambda \mathbf{I} & \mathbf{B}_{I}
\end{array}\right]=n-d_{I}
$$

where $d_{I}$ is a nonnegative integer. Thus, the matrix $\left[\mathbf{E}_{F}(\mathbf{W}-\lambda \mathbf{I})^{-1} \mathbf{H C} \mathbf{0}\right]$ must provide $d_{I}$ rows that are linearly independent of all rows in $\left[\begin{array}{ll}\mathbf{A}-\lambda \mathbf{I} & \mathbf{B}_{I}\end{array}\right]$. We will provide conditions on the WCN topology to guarantee this.

First, due to the assumption that the pair $(\mathbf{A}, \mathbf{C})$ is detectable, we have rank $\left[\begin{array}{c}\mathbf{A}-\lambda \mathbf{I} \\ \mathbf{C}\end{array}\right]=n$ for any unstable eigenvalue $\lambda$ of $\mathbf{A}$. This means that for any set $I \subseteq \mathcal{M}$, there are $d_{I}$ rows in the matrix $\left[\begin{array}{ll}\mathbf{C} & \mathbf{0}\end{array}\right]$ that are linearly independent of the rows in $\left[\begin{array}{ll}\mathbf{A}-\lambda \mathbf{I} & \mathbf{B}_{I}\end{array}\right]$. Let $J_{1}^{\prime}, J_{2}^{\prime}, \ldots, J_{s}^{\prime}$ be all possible sets of $d_{I}$ rows of $\mathbf{C}$ that satisfy this linear independence property, and let $\mathcal{Y}_{1}, \mathcal{Y}_{2}, \ldots, \mathcal{Y}_{s}$ be the sets of $d_{I}$ outputs of the plant corresponding to those rows. If we can guarantee that the row space of $\mathbf{C}_{J_{i}^{\prime}}$ is contained in the row space of $\mathbf{E}_{F}(\mathbf{W}-\lambda \mathbf{I})^{-1} \mathbf{H C}$ for some $i$, then the right hand side of (15) will be at least $N+n$.

To satisfy this condition, note that $\mathbf{E}_{F}(\mathbf{W}-\lambda \mathbf{I})^{-1} \mathbf{H}$ in (15) is the transfer function of the WCN (where the outputs are taken to be nodes in the set $\mathcal{V}_{\mathcal{M} \backslash I}$ ) evaluated at $\lambda$. This matrix must have rank at least $d_{I}$ in order for the right hand side of (15) to have rank $n$. To analyze this condition, we can consider a general structured linear system $\Sigma$, and ask what the largest possible rank of the transfer function would be over all possible values of the nonzero free parameters and $\lambda$; this is called the generic rank of the transfer function matrix for the system. The following result from [17] relates this rank to a property of the graph associated with the system.

Theorem 6 ([17]): Let $\Sigma=(\mathbf{A}, \mathbf{B}, \mathbf{C})$ be a structured linear system, and let $\mathcal{G}_{\Sigma}$ be its associated graph. Then, the

\footnotetext{
${ }^{13}$ For a structured square matrix $\mathbf{W}$ and for a finite set of nonzero complex numbers $\mathcal{L}$, the eigenvalues of $\mathbf{W}$ will all be different from the elements of $\mathcal{L}$ for almost any choice of parameters in $\mathbf{W}$ [8].
}

generic rank of the transfer function matrix is equal to the size of the largest linking from the input vertices to the output vertices in $\mathcal{G}_{\Sigma}$.

We now present a result guaranteeing that the transfer function matrix will be full rank when evaluated at certain values $\lambda$.

Lemma 2: Consider the structured system $\Sigma=$ $(\mathbf{A}, \mathbf{B}, \mathbf{C})$, and suppose that the graph $\mathcal{G}_{\Sigma}$ contains a linking of size $m$ from the input vertices to the output vertices. Let $\mathcal{L}=\left\{\lambda_{1}, \lambda_{2}, \ldots, \lambda_{r}\right\}$ be a finite set of nonzero complex numbers. Then,

$$
\operatorname{rank}\left(\mathbf{C}\left(\mathbf{A}-\lambda_{i} \mathbf{I}\right)^{-1} \mathbf{B}\right)=m, \quad i \in\{1,2, \ldots, r\},
$$

for almost any choice of free parameters in $(\mathbf{A}, \mathbf{B}, \mathbf{C})$.

The proof is conceptually similar to the proof of Lemma 1.48 in [8], and we omit it here in the interest of space. For a detailed proof, see [15].

Now that we have a handle on some rank properties of the matrix $\mathbf{E}_{F}(\mathbf{W}-\lambda \mathbf{I})^{-1} \mathbf{H}$, we return to the problem of ensuring that the row space of $\mathbf{C}_{J_{i}^{\prime}}$ is contained in the row space of $\mathbf{E}_{F}(\mathbf{W}-\lambda \mathbf{I})^{-1} \mathbf{H C}$, for some $i \in\{1,2, \ldots, s\}$. The following theorem provides topological conditions for the WCN to satisfy to guarantee that this condition holds.

Theorem 7: Consider the detectable and stabilizable (numerical) system $\Sigma=(\mathbf{A}, \mathbf{B}, \mathbf{C})$, along with a WCN. Let $\lambda$ be an unstable eigenvalue of $\mathbf{A}$. For any subset $I \subseteq \mathcal{M}$, let $d_{I}=n-\operatorname{rank}\left[\begin{array}{ll}\mathbf{A}-\lambda \mathbf{I} & \mathbf{B}_{I}\end{array}\right]$. If for every possible subset $I$, there exists a subset $J^{\prime}$ of $d_{I}$ plant outputs such that $\operatorname{rank}\left[\begin{array}{cc}\mathbf{A}-\lambda \mathbf{I} & \mathbf{B}_{I} \\ \mathbf{C}_{J^{\prime}} & \mathbf{0}\end{array}\right]=n$, and the $\mathrm{WCN}$ contains a $d_{I}$ linking from those outputs to $\mathcal{V}_{\mathcal{M} \backslash I}$, then for almost any choice of free parameters in $\mathbf{W}$ and $\mathbf{H}, \lambda$ is not a fixed mode of the system $\tilde{\Sigma}$. Furthermore, if the above holds for every unstable eigenvalue of $\mathbf{A}$, then for almost any choice of parameters in $\mathbf{W}$ and $\mathbf{H}$ such that $\mathbf{W}$ is a stable matrix, system $\tilde{\Sigma}$ will have no unstable fixed modes.

Proof: For a given subset $I$ and corresponding set $\mathcal{V}_{\mathcal{M} \backslash I}$, denote the graph of the structured system $\Sigma_{W C N}=$ $\left(\mathbf{W}, \mathbf{H}, \mathbf{E}_{F}\right)$ by $\mathcal{G}_{\Sigma_{W C N}}$. Noting that the inputs to the WCN are the outputs of the plant, the input vertices in $\mathcal{G}_{\Sigma_{W C N}}$ are given by $\mathcal{Y}$. Furthermore, denote the output vertices of $\mathcal{G}_{\Sigma_{W C N}}$ by $\mathcal{V}_{\mathcal{M} \backslash I}$. Consider any subset $I \subseteq \mathcal{M}$ for which $d_{I}>0$, and let $\mathcal{Y}^{\prime}$ be the set of $d_{I}$ outputs corresponding to the set $J^{\prime}$ described in the theorem. According to the assumption in the theorem, the graph $\mathcal{G}_{\Sigma_{W C N}}$ contains a linking of size $d_{I}$ from these outputs to $\mathcal{V}_{\mathcal{M} \backslash I}$. Let $\mathbf{H}_{J^{\prime}}$ denote the matrix consisting of the columns of $\mathbf{H}$ corresponding to the outputs in set $\mathcal{Y}^{\prime}$, and consider the system $\left(\mathbf{W}, \mathbf{H}_{J^{\prime}}, \mathbf{E}_{F}\right)$. The graph of this system is obtained simply by removing the vertices that are not in $\mathcal{Y}^{\prime}$ from the graph $\mathcal{G}_{\Sigma_{W C N}}$. Since this reduced graph has an $d_{I}$-linking from the inputs to the outputs, Theorem 6 and Lemma 2 indicate that $\mathbf{E}_{F}(\mathbf{W}-\lambda \mathbf{I})^{-1} \mathbf{H}_{J^{\prime}}$ will have rank $d_{I}$ for almost choice of free parameters in $\mathbf{W}$ and $\mathbf{H}_{J^{\prime}}$. Thus, $\mathbf{E}_{F}(\mathbf{W}-\lambda \mathbf{I})^{-1} \mathbf{H}_{J^{\prime}} \mathbf{C}_{J^{\prime}}$ will have rank $d_{I}$, and

$$
\operatorname{rank}\left[\begin{array}{cc}
\mathbf{A}-\lambda \mathbf{I} & \mathbf{B}_{I} \\
\mathbf{E}_{F}(\mathbf{W}-\lambda \mathbf{I})^{-1} \mathbf{H}_{J^{\prime}} \mathbf{C}_{J^{\prime}} & \mathbf{0}
\end{array}\right]=n
$$

Now, consider the matrix $\left[\begin{array}{cc}\mathbf{A}-\lambda \mathbf{I} & \mathbf{B}_{I} \\ \mathbf{E}_{F}(\mathbf{W}-\lambda \mathbf{I})^{-1} \mathbf{H C} & \mathbf{0}\end{array}\right]$; this matrix has rank $n$ for some choice of $\mathbf{W}$ and $\mathbf{H}$ (i.e., by setting 
the columns of $\mathbf{H}$ that are not in $J^{\prime}$ to be zero, and choosing all other parameters almost arbitrarily). Thus, there is an $n$ th order minor of the above matrix that is nonzero. Now, setting all nonzero entries in the columns of $\mathbf{H}$ that are not in $\mathbf{H}_{J^{\prime}}$ to be free parameters, this minor will be a nonzero polynomial in those parameters. Thus, the set of parameters for which the rank of the above matrix is less than $n$ lies on an algebraic variety, and so the above matrix has rank at least $n$ for almost any choice of free parameters.

The above analysis holds for every subset $I \subseteq \mathcal{M}$, and thus $\lambda$ is not a fixed mode of the system; furthermore, if the conditions in the theorem hold for every unstable eigenvalue of $\mathbf{A}$, all of these eigenvalues will not be fixed modes for almost any choice of free parameters in $\mathbf{W}$ and $\mathbf{H}$. Finally, note that the eigenvalues of $\tilde{\mathbf{A}}$ in (12) are the union of the eigenvalues of $\mathbf{A}$ and $\mathbf{W}$. The set of free parameters that makes $\mathbf{W}$ a stable matrix has measure greater than zero, and the above analysis will hold for almost any such choice of parameters. Furthermore, this guarantees that no eigenvalue of $\mathbf{W}$ can be an unstable fixed mode, which concludes the proof.

While the above result provides a method to test if the system has any fixed modes when controlled over a WCN, it is rather cumbersome to apply because of the need to test all possible subsets of $\mathcal{M}$. The following result provides a sufficient condition for the system to have no fixed modes that is much easier to check and enforce.

Theorem 8: Consider the detectable and stabilizable system $\Sigma=(\mathbf{A}, \mathbf{B}, \mathbf{C})$, along with a WCN. Let $d$ denote the largest geometric multiplicity of any unstable eigenvalue of A. Suppose the connectivity of the network is at least $d$, and each actuator has at least $d \mathrm{WCN}$ nodes in its neighborhood. Then, for almost any choice of parameters in $\mathbf{W}$ and $\mathbf{H}$ such that $\mathbf{W}$ is stable, the system $\tilde{\Sigma}$ can be stabilized via a dynamic compensator at each actuator.

Proof: First, note that for any unstable eigenvalue $\lambda$ of $\mathbf{A}$, we have $\operatorname{rank}(\mathbf{A}-\lambda \mathbf{I}) \geq n-d$, and thus the quantity $d_{I}$ specified in Theorem 7 is no larger than $d$. Also, for any subset $I \subseteq \mathcal{M}$, let $J^{\prime}$ be the set of $d_{I}$ outputs specified in that theorem, let $\mathcal{Y}^{\prime}$ be the corresponding set of outputs, and let $\mathcal{V}_{S}^{\prime}$ be the nodes in the WCN that receive information from the outputs in $\mathcal{Y}^{\prime}$. Next, note that $\left|\mathcal{V}_{\mathcal{M} \backslash I}\right| \geq d \geq d_{I}$ by the assumption that each actuator has at least $d$ wireless nodes in its neighborhood. Since the connectivity of the network is $d$, and since $\left|\mathcal{V}_{\mathcal{M} \backslash I}\right| \geq d$ and $\left|\mathcal{V}_{S}^{\prime}\right|=\left|\mathcal{Y}^{\prime}\right|=d_{I} \leq d$ (by the assumption in Footnote 9), there exists a linking of size $d_{I}$ from the set $\mathcal{V}_{S}^{\prime}$ to $\mathcal{V}_{\mathcal{M} \backslash I}$ [18]. Thus, all conditions in Theorem 7 are satisfied, and the system will have no unstable fixed modes.

\section{CONCLUSION AND DISCUSSION}

In this paper we have presented a set of topological conditions for a WCN to satisfy in order to stabilize both structured and numerically specified plants. Our analysis shows that as long as these conditions are satisfied, the system will not have fixed modes, and can be stabilized via a dynamic compensator at each actuator. An interesting byproduct of our analysis is the following observation: the network diameter does not enter into the conditions required for stabilization over the WCN. In other words, delays in the network are not a factor when considering the issue of stability. This is achieved by incorporating dynamics into the network via the linear iterative strategy, and then designing the controllers to capture the dynamics of the plant and network simultaneously. However, while stability is guaranteed despite the lengths of the paths in the network (as long as the network satisfies the appropriate connectivity requirements specified in Theorem 3 or Theorem 8), we conjecture that the robustness or performance of the closedloop system will potentially suffer. A detailed analysis of this phenomenon will be the subject of future research.

\section{REFERENCES}

[1] O. C. Imer, S. Yuksel, and T. Basar, "Optimal control of LTI systems over unreliable communication links," Automatica, vol. 42, no. 9, pp. 1429-1439, Sep. 2006.

[2] J. P. Hespanha, P. Naghshtabrizi, and Y. Xu, "A survey of recent results in networked control systems," Proceedings of the IEEE, vol. 95, no. 1, pp. 138-162, Jan. 2007.

[3] L. Schenato, B. Sinopoli, M. Franceschetti, K. Poolla, and S. S. Sastry, "Foundations of control and estimation over lossy networks," Proc. of the IEEE, vol. 95, pp. 163-187, 2007.

[4] V. Gupta, A. F. Dana, J. Hespanha, R. M. Murray, and B. Hassibi, "Data transmission over networks for estimation and control," IEEE Transactions on Automatic Control, vol. 54, no. 8, pp. 1807-1819, Aug. 2009.

[5] M. Pajic, S. Sundaram, R. Mangharam, and G. J. Pappas, "The Wireless Control Network: A New Approach for Control over Networks," IEEE Transactions on Automatic Control, 2011, To appear.

[6] S.-H. Wang and E. Davison, "On the stabilization of decentralized control systems," IEEE Transactions on Automatic Control, vol. 18, no. 5, pp. 473-478, 1973.

[7] M. Sezer and D. Siljak, "Structurally fixed modes," Systems \& Control Letters, vol. 1, no. 1, pp. 60-64, 1981.

[8] D. Siljak, Decentralized control of complex systems. Academic Press, Inc, 1991.

[9] J. Lavaei and S. Sojoudi, "Time complexity of decentralized fixed-mode verification," IEEE Transactions on Automatic Control, vol. 55, no. 4, pp. 971-976, Apr. 2010.

[10] J.-M. Dion, C. Commault, and J. van der Woude, "Generic properties and control of linear structured systems: a survey," Automatica, vol. 39, no. 7, pp. 1125-1144, July 2003.

[11] K. J. Reinschke, Multivariable Control A Graph-Theoretic Approach. Springer-Verlag, 1988.

[12] V. Pichai, M. E. Sezer, and D. D. Siljak, "A graph-theoretic characterization of structurally fixed modes," Automatica, vol. 20, no. 2, pp. 247-250, 1984.

[13] L. El Ghaoui, F. Oustry, and M. Ait Rami, "A cone complementarity linearization algorithm for static output-feedback and related problems," IEEE Transactions on Automatic Control, vol. 42, no. 8, pp. 1171-1176, Aug. 1997.

[14] J. L. Willems, "Time-varying feedback for the stabilization of fixed modes in decentralized control systems," Automatica, vol. 25, no. 1, pp. 127-131, 1989.

[15] M. Pajic, S. Sundaram, R. Mangharam, and G. J. Pappas, "The Wireless Control Network: Topological Conditions for System Stabilization," Technical Report, 2011.

[16] M. E. Sezer, "Minimal essential feedback patterns for pole assignment using dynamic compensation," in Proc. of the 22nd IEEE Conference on Decision and Control, 1983, pp. 28-32.

[17] J. W. van der Woude, "A graph-theoretic characterization for the rank of the transfer matrix of a structured system," Mathematics of Control, Signals and Systems, vol. 4, no. 1, pp. 33-40, Mar. 1991.

[18] D. B. West, Introduction to Graph Theory. Prentice-Hall Inc., Upper Saddle River, New Jersey, 2001. 